\section{Test of a multidimensional discrimination model of stimulus identification*}

\author{
ROBERT M. LEVY† and STEVEN J. HAGGBLOOM \\ Indiana State University, Terre Haute, Ind. 47809
}

Lockhead's hypothesis that distribution in psychological space determines the identifiability of multidimensional stimuli was tested using both redundant and nonredundant stimulus sets. Three four-item bidimensional stimulus sets were used. In order of increasing aistribution in psychological space, they were: redundant linear correlated, redundant sawtooth, and nonredundant. Fifteen Ss were run, five per condition, for 96 test trials in each of four sessions. As predicted, both amount and rate of information transmission were greatest for the nonredundant stimuli; however, there were no significant differences between the redundant sets.

Lockhead (1970) recently suggested that the identifiability of a set of multidimensional stimuli is primarily determined by their distribution in a "psychological space" independent of the redundancy of the values on component dimensions. Given two sets of bidimensional redundant stimuli with the same number of alternatives, the greater the distances among the items in the "multidimensional discrimination space," the more identifiable the stimuli within the set.

The bidimensional grid in Fig. 1 represents "psychological space" and the distribution within this space of two bidimensional stimulus sets with each dimension having four values. Lockhead considered two bidimensional pairings: linear correlated, shown with $\times \mathbf{s}$, and what he termed "sawtooth," shown with -s. The stimuli in both sets are redundant, i.e., the value on one dimension can be predicted perfectly by knowing the value on the other dimension; however, in the psychological space, the distances between the sawtooth stimuli are greater than the distances between the linear correlated stimuli. As he predicted, Lockhead found that more information was transmitted with the sawtooth combinations. If the distribution in psychological space and not redundancy determines identifiability, as Lockhead (1970) argued, then we would also predict that the stimuli indicated with $=\mathrm{s}$ in the four corners of the grid in Fig. 1 would constitute the most identifiable stimulus set. A noteworthy feature of this set is that the stimuli are nonredundant, i.e., the value of $B$ cannot be predicted from the value of A.

* The writers wish to thank Ann Lovett and Darlene Wilke for their assistance in collecting the data.

-Requests for reprints should be addressed to Robert M. Levy, Department of Psychology. Indiana State Lniversity. Terre Haute. Indiand 47809 .
The purpose of the present experiment was to test Lockhead's model by comparing identification performance on three bidimensional stimulus conditions: redundant linear correlated, redundant sawtooth, and nonredundant. Both speed and accuracy of identification were measured to provide as complete a description of information processing as possible.

\section{EXPERIMENTAL CONDITIONS} AND APPARATUS

The stimuli, mounted on white tagboard, were square patches of Munsell paper which varied in both size $(1.00,1.32,1.75$, and $2.31 \mathrm{sq} \mathrm{cm})$ and brightness (N6.5/, N6), N5.5/, $\mathrm{N} 5 /$ ), designated as $\mathrm{S}_{1}, \mathrm{~S}_{2}, \mathrm{~S}_{3}, \mathrm{~S}_{4}$, and $B_{1}, B_{2}, B_{3}, B_{4}$, respectively. The three sets of four bidimensional stimuli were: redundant linear correlated, $\mathrm{RC}$, $\mathrm{S}_{1} \mathrm{~B}_{1}, \mathrm{~S}_{2} \mathrm{~B}_{2}, \mathrm{~S}_{3} \mathrm{~B}_{3}, \mathrm{~S}_{4} \mathrm{~B}_{4}$; redundant sawtooth, RS, $\mathrm{S}_{2} \mathrm{~B}_{1}, \mathrm{~S}_{4} \mathrm{~B}_{2}, \mathrm{~S}_{3} \mathrm{~B}_{4}$, $S_{1} B_{3}$; and nonredundant, $N R, S_{1} B_{1}$, $\mathrm{S}_{1} \mathrm{~B}_{4}, \mathrm{~S}_{4} \mathrm{~B}_{4}, \mathrm{~S}_{4} \mathrm{~B}_{1}$. The responses, assigned to the stimuli in the order presented above, were "one," "two," "three," "four," with the stop consonant " $b$ " prefixed in order to control voicing time for the noise-operated relay, i.e., "bun," "boo," "bee," "bore."

All stimuli were centered in the $73 / 4 \times 73 / 4 \mathrm{in}$. viewing field of a Gerbrands Model T-2B-1 two-channel tachistoscope. The stimulus field, $21 \mathrm{in}$. from the $S$, and the blank preand postexposure fields were of equal subjective luminance. A Hunter Millisecond Klockounter started with the initiation of each stimulus exposure, and the S's vocal response activated a Hunter noise relay which stopped the Klockounter and terminated the exposure.

\section{SUBJECTS}

Fifteen undergraduate students participated in the experiment to fulfill a course requirement. Each $S$ was run individually for four $1 / 2-h$ sessions on different days. Five $\mathrm{Ss}$ were assigned randomly to each of the three experimental conditions. PROCEDURE

At the beginning of the first session, the appropriate stimuli were shown to the $S$ outside of the tachistoscope and named. The stimuli were then shown a few times inside the tachistoscope, and the $\mathrm{S}$ was asked to identify them.

When the $\mathbf{S}$ depressed a hand-held pushbutton, the tachistoscope's preexposure field was switched to the stimulus field and the clock started. The onset of the S's vocal response activated the noise relay, which switched the tachistoscope from the stimulus to the postexposure field and stopped the clock. Following each trial, the $S$ was told the correct response and the choice reaction time, CRT. There were 24 practice and 96 test trials in each session, with the order of stimulus presentation randomized with the constraint that the four stimuli occur equally often. The $\mathbf{S}$ was asked to respond as quickly and accurately as possible. The instructions were reviewed at the beginning of each session.

\section{RESULTS AND DISCUSSION}

The amount of information transmitted, $T(S: R)$, and the median CRT were calculated for each $\mathrm{S}$ for the last 96 trials of each session. In addition, the rate of information transmission, $\dot{T}(S: R)$, was estimated from the ratio $\mathrm{T}(\mathrm{S}: \mathrm{R}) / \mathrm{CRT}$, Figure 2 shows $T(S: R), C R T$, and $T(S: R)$ as functions of experimental condition and session.

Contrary to predictions from Lockhead's (1970) model, the identifiability in the RS condition was not significantly better than that in the RC condition as measured by $\mathrm{T}(\mathrm{S}: \mathrm{R})$, CRT, or $\mathbf{T}(\mathrm{S}: \mathrm{R})$. However, there was a significant interaction on CRT between the redundant sets from

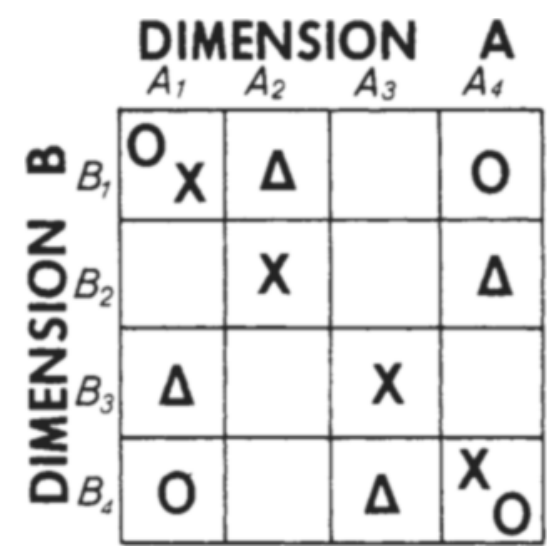

Fig. 1. Distribution of linear correlated redundant $(X s)$, sawtooth redundant $(-s)$, and nonredundant (is) stimulus sets in hypothetical psychological space. 


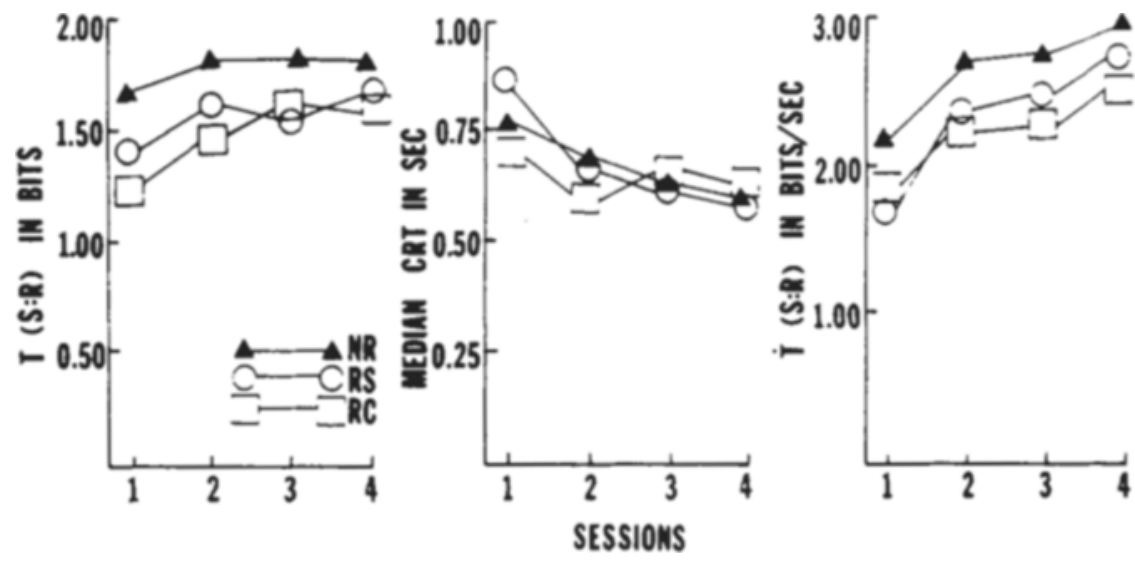

Fig. 2. Amount of information transmitted (left panel), median choice reaction time (center panel), and rate of information transmission (right panel) as functions of experimental condition and session.

Session 1 to Sessions 2,3 , and 4 $[F(1,36)=10.35, p<.01]$, with the CRTs in Session 1 originally much slower for the RS condition. This may reflect differences in S-R compatibility (cf. Fitts \& Seeger, 1953), since the RS stimuli do not preserve the natural ordering present, by definition, in the correlated component values of the RC stimuli. Alternatively, the increased CRT for the RS stimuli during Sessions 1 and 2 may indicate a trade-off between speed and accuracy, since more information was transmitted in the RS condition than in the RC condition, as can be seen in the first graph in Fig. 1. During Sessions 1 and 2 , there was negligible difference in $T(S: R)$ between the two conditions; however, Lockhead's model is partially supported by the greater rate over the last two sessions for the RS condition.

Comparison of the NR against the pooled $\mathrm{RC}$ and $\mathrm{RS}$ conditions showed that significantly more information was transmitted $[F(1,12)=11.875$ $\mathrm{p}<.01]$ and at a higher rate $[F(1,12)=8.736, p<.025]$ in the NR condition. The clear-cut superiority of the NR condition as measured by both amount and rate of information transmitted supports the hypothesis that the major determinant of the distinctiveness of a set of multidimensional stimuli is their distribution in psychological space, independent of redundancy. Lockhead (1970) used redundant stimuli; the present experiment extended the model to include nonredundant stimuli.
For the NR set used in the present experiment, there were three steps between adjacent values on each dimension compared to the one step between values in the $R S$ and $R C$ conditions, i.e., the intradimensional discriminability was confounded between the redundant and nonredundant conditions. Furthermore, not only is the distribution of stimuli in psychological space assumed to be greatest in the NR condition, it is also true that the number of values on each dimension i.e., the dimensional load, is reduced from four to two. The potential significance of the dimensional load factor can be better appreciated if one considers a set of 16 bidimensional stimuli. In this case, the NR sets would have four values on each dimension, whereas the RS and RC conditions would have 16 values on each dimension, which clearly exceeds the dimensional capacity Miller (1956) discussed. Additional research is presently under way to investigate the roles of intradimensional discriminability and dimensional load in multidimensional stimulus identification.

\section{REFERENCES}

FITTS, P. M., \& SEEGER, C. H. S-R compatibility: Spatial characteristics of stimulus and response codes. Journal of Experimental Psychology, 1953, 46. 199-210.

LOCKHEAD, G. R. Identification and the form of multidimensional discrimination space. Journal of Experimental Psychology, $1970,85,1-10$.

MILLER. G. A. The magical number seven, plus or minus two. Psychological Review, $1956,63,81-97$. 